\title{
Solvent-Assisted Self-Assembly of Gold Nanorods into Hierarchically Organized Plasmonic Mesostructures
}

\author{
Christoph Hanske, ${ }^{* \dagger}{ }^{\dagger}$ Eric H. Hill, ${ }^{\dagger, \|}$ David Vila-Liarte, ${ }^{\dagger}$ Guillermo González-Rubio, ${ }^{\dagger}$ \\ Cristiano Matricardi, ${ }^{\ddagger}$ Agustín Mihi, ${ }^{\ddagger}$ and Luis M. Liz-Marzán*, ${ }^{*}, \S_{(0)}$ \\ ${ }^{\dagger}$ CIC biomaGUNE and Ciber-BBN, Paseo de Miramón 182, 20014 Donostia-San Sebastián, Spain \\ ${ }^{\ddagger}$ Institut de Ciència de Materials de Barcelona (ICMAB-CSIC), Campus de la UAB, 08193 Bellaterra, Catalonia, Spain \\ ${ }^{\S}$ Ikerbasque, Basque Foundation of Science, 48013 Bilbao, Spain
}

\section{Supporting Information}

\begin{abstract}
Plasmonic supercrystals and periodically structured arrays comprise a class of materials with unique optical properties that result from the interplay of plasmon resonances, as well as near- and far-field coupling. Controlled synthesis of such hierarchical structures remains a fundamental challenge, as it demands strict control over the assembly morphology, array size, lateral spacing, and macroscale homogeneity. Current fabrication approaches involve complicated multistep procedures lacking scalability and reproducibility, which has hindered the practical application of plasmonic supercrystal arrays. Herein, these challenges are

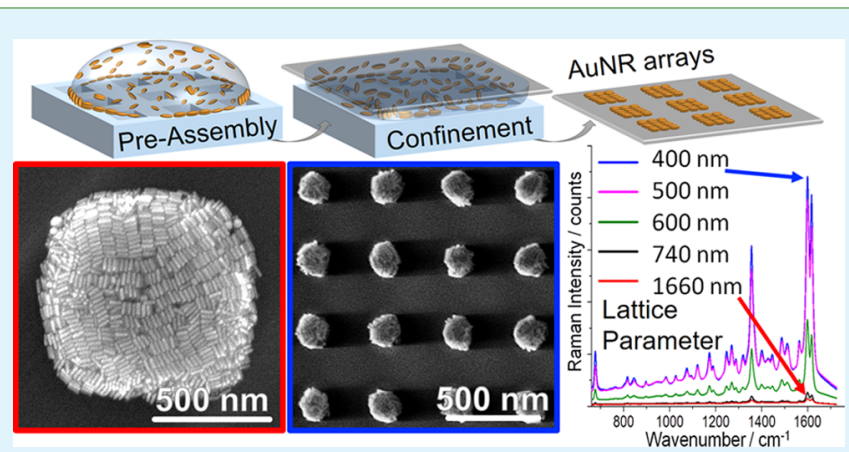
addressed by adding an organic solvent to achieve kinetic control over the template-assisted colloidal assembly of nanoparticles from aqueous dispersion. This method yields highly regular periodic arrays, with feature sizes ranging from less than $200 \mathrm{~nm}$ up to tens of microns. A combined experimental/ computational approach reveals that the underlying mechanism is a combination of the removal of interfacial surfactant micelles from the particle interface and altered capillary flows. Assessing the efficacy of such square arrays for surface-enhanced Raman scattering spectroscopy, we find that a decrease of the lattice periodicity from $750 \mathrm{~nm}$ down to $400 \mathrm{~nm}$ boosts the signal by more than an order of magnitude, thereby enabling sensitive detection of analytes, such as the bacterial quorum sensing molecule pyocyanin, even in complex biological media.
\end{abstract}

KEYWORDS: template-assisted self-assembly, gold nanorods, SERS, lattice plasmon, molecular dynamics simulation

\section{INTRODUCTION}

Due to their strong interaction with electromagnetic radiation, microarrays of plasmonic nanoparticles show great promise as components for high-performance optical sensors, light managing layers in thin-film solar cells, novel color displays, and other applications. ${ }^{1-6}$ In this context, tight control over the array dimensions, internal structure, and overall macroscopic homogeneity are crucial. The fabrication of plasmonic mesostructures (i.e., structures with feature sizes ranging from $100 \mathrm{~nm}$ to microns) with controlled properties still poses a significant challenge, as it requires bridging vastly different length scales, typically ranging from few nanometers up to several millimeters, for technological feasibility.

Arrangements of noble-metal nanoparticles are the most common class of plasmonic nanomaterials for the fabrication of optical sensors, employed, for example, in surface-enhanced Raman scattering (SERS) or surface-enhanced infrared absorption (SEIRA) spectroscopy. ${ }^{3,8}$ These applications rely heavily on strong light-matter interactions in the resonant noble-metal structure carrying the analyte to be detected. $3,9,10$ Gold nanoparticles are often the material of choice, as their stability against oxidation grants easy fabrication of clean surfaces that can get into direct contact with analyte molecules. For biosensing in particular, gold displays further advantages due to its low cytotoxicity, which can be problematic when using other plasmonic nanomaterials such as silver or copper, for measurements in complex media or even in living systems. Among the many accessible shapes, anisotropic nanoparticles such as gold nanorods (AuNRs) excel due to the straightforward tunability of their localized surface plasmon resonances via chemical adjustment of the nanoparticles' aspect ratio. $^{11,12}$ This geometrical control allows precise tailoring of the desired excitation wavelength and even achieving optical monodispersity. ${ }^{13}$ Further signal enhancement, needed for chemical ultradetection or even single molecule detection, requires optimization of near-field and farfield coupling effects to boost light-matter interactions. A strong near-field coupling relies on interparticle distances of

Received: January 7, 2019

Accepted: March 7, 2019

Published: March 7, 2019 
$1-2 \mathrm{~nm},{ }^{14-18}$ which in practice rules out purely top-down approaches such as e-beam lithography or focused ion beam milling, which are just about to reach gap dimensions below 5 nm. ${ }^{19}$ Far-field coupling, on the other hand, depends on diffraction and requires periodic arrays with close-to-perfect registration over extended areas. ${ }^{20}$ As a result, templateassisted self-assembly of noble-metal colloids is the currently preferred fabrication strategy, which efficiently combines both near-field and far-field coupling, to take full advantage of optical enhancement. ${ }^{21,22}$

For biomolecule detection, the ability to identify specific substances within complex mixtures (often at submicromolar concentrations) is of key importance and has fueled research in the field of SERS. Recent highlights include the detection of prostate-specific antigens in serum, multiplexed cancer type discrimination in cell cultures, and even SERS-based in vivo imaging of tumors. ${ }^{23}$ Self-assembled AuNR structures in particular have been used for the detection of scrambled prions or pyocyanin (a bacterial quorum-sensing molecule expressed by the human pathogen Pseudomonas aeruginosa) via SERS. $^{24,25}$ The supercrystals used in these examples exhibit strong plasmonic near-field coupling due to the nanometersized interparticle distances, thereby allowing molecular detection in vitro in complex biological media, or even in situ in live bacterial cell cultures. However, in these examples, the nanoparticles were assembled into isolated supercrystals or micrometer-sized structures with array periodicities around 10 $\mu \mathrm{m}$, and thus did not take advantage of far-field coupling. As shown by Reinhard et al. for micrometer-sized arrays, near-field coupled colloidal clusters can deliver significantly amplified signal outputs when arranged in a periodic lattice. ${ }^{26}$ The farfield modes of the array must therefore be in resonance with the exciting light source, usually demanding submicrometer lattice periodicities. These so-called lattice plasmons comprise an attractive route toward SERS substrates that offer high sensitivity with low noble metal consumption and a reduced

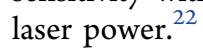

Despite recent reports on the colloidal assembly of AuNRs into micrometer-sized supercrystals, the production of highquality AuNR arrays with submicrometer feature sizes has proven to be an extraordinarily elusive task. ${ }^{27}$ Most of the current strategies are complicated, multistep procedures based on pre-assembly by spin or dip coating, followed by transfer printing onto a target substrate. ${ }^{28,29}$ In fact, there is little understanding of the factors influencing macroscopic array homogeneity, which include particle surface functionalization, residual surfactant, and wetting of both the stamp and the target substrate. Thus, the scaling and reproducibility of such assembly methods remain problematic. Recently, regular AuNR arrangements have been realized by combining topographically structured templates with controlled convective assembly or electrophoretic deposition techniques. $^{30-32}$ However, to the best of our knowledge, no fast, scalable method is available that yields homogeneous arrays of regular AuNR mesostructures over macroscopic surface areas, with no need for sophisticated equipment.

We present herein an optimized approach for the selfassembly of gold nanorods (length: $55 \pm 5 \mathrm{~nm}$, diameter: 16.5 $\pm 1.5 \mathrm{~nm}$ ) coated with (11-mercaptoundecyl)hexa(ethylene glycol) (MUHEG) into highly regular structures, using ethanol/water mixtures as the dispersion media. Functionalization with MUHEG has been shown to foster side-by-side organization of nanorods into structures with feature sizes between 1 and $50 \mu \mathrm{m}$ for assembly from aqueous dispersions. ${ }^{27}$ In this work, much smaller, hierarchically structured plasmonic arrays with feature sizes ranging from $200 \mathrm{~nm}$ up to $10 \mu \mathrm{m}$ and periodicities from $400 \mathrm{~nm}$ to $20 \mu \mathrm{m}$ are realized by a solvent-assisted approach. Through systematic optimization of solvent composition and particle and surfactant concentrations, as well as a detailed investigation of the role of substrate application timing, mesostructured AuNR arrays with unprecedented quality are achieved over areas as large as 0.5 $\mathrm{cm}^{2}$. All-atom molecular dynamic (MD) simulations are used to elucidate the role of the volatile solvent (ethanol) in improving the nanoparticle self-assembly, which is experimentally confirmed by $\zeta$-potential measurements. Ethanol is shown to strip away excess cetyltrimethylammonium bromide (CTAB) micelles from the MUHEG/water interface, thereby increasing the hydrophobicity of the particles and triggering reversible agglomeration. We thus propose an assembly mechanism that takes into account the preorganization of nanoparticles at the liquid/gas interface, which is further confirmed by a kinetic study via photomicroscopy of drying droplets and correlated atomic force microscopy (AFM)/ scanning electron microscopy (SEM) characterization of the resulting substrates. Finally, application of the obtained plasmonic materials as SERS sensors for biomolecule detection is demonstrated both in water and in complex biological media.

\section{RESULTS AND DISCUSSION}

Template-assisted nanoparticle deposition has recently become a promising nanofabrication method that combines bottom-up and top-down approaches. The scalability of this method has been largely facilitated by the synthesis of colloidal nanoparticles in various shapes, with high monodispersity, ${ }^{33-35}$ along with simple and inexpensive production of microstructured stamps by soft lithography. ${ }^{16,36,37}$ However, the quality of the nanomaterial is often compromised because of the well-known coffee ring effect: inhomogeneous material distribution (in the worst case, accumulation outside of the structured area) is observed when the dispersed nanoparticles are driven toward the rim of the confined droplet during drying due to convective forces (Figure 1a). ${ }^{38}$ We recently reported that template-assisted assembly of sterically stabilized (i.e., poly(ethylene glycol)-capped) gold nanospheres (PEGAuNSs) improved when the amount of residual surfactant cetyltrimethylammonium chloride (CTAC) in the dispersion was kept far below its critical micellar concentration ( $\mathrm{cmc}$ of CTAC: $1.6 \mathrm{mM}$ at $\left.25{ }^{\circ} \mathrm{C}\right) .{ }^{39}$ The most homogeneous structures were achieved with a surfactant concentration of around $50 \mu \mathrm{M}$, at which the nanospheres would slowly agglomerate in the dispersion, forming extended golden films on the hydrophobic walls of plastic storage vessels. ${ }^{22}$ For the MUHEG-coated rods used in this study, we could neither replicate the described effects nor sufficiently suppress the dominant coffee ring effect by simply decreasing the concentration of free surfactant, which was tested down to $30 \mu \mathrm{M}$ residual surfactant (see Figures $1 \mathrm{~b}$ and S2). To improve the wetting of the polydimethylsiloxane (PDMS) stamp, ${ }^{40}$ ethanol (EtOH) was added to the dispersions, thereby enabling a nearly complete suppression of the coffee ring formation, which had strongly hindered the fabrication of welldefined AuNR arrays over areas larger than a few hundred $\mu \mathrm{m}^{2}{ }^{27}$ Unexpectedly, the presence of EtOH also changed the 


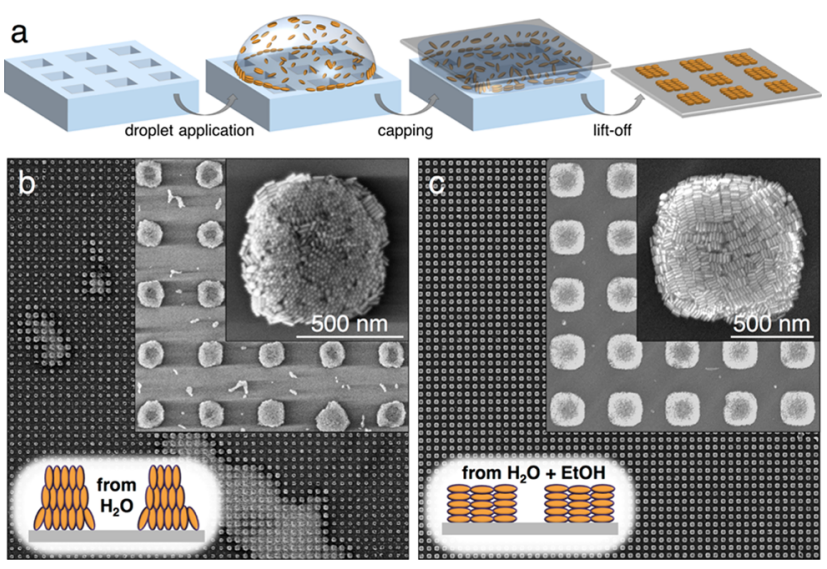

Figure 1. Comparison of MUHEG-AuNR assembly from water vs $\mathrm{EtOH} /$ water mixtures. (a) Scheme of the patterning process, which comprised drying of a dispersion droplet confined between a structured soft PDMS stamp and a flat target substrate. (b) SEM images of the best microarrays that could be obtained from MUHEGcoated AuNRs dispersed in water. (c) Representative SEM images from an arbitrary part of a typical substrate obtained with the same particles but now dispersed in an EtOH/water mixture. Only with ethanolic dispersions, large area pattern replication was possible, and highly regular arrays of lying rod lamellae were formed.

orientation of the nanorods from standing rods into side-byside packed lamellae, as shown in Figures 1c and S3.

Intriguingly, ethanol-containing AuNR dispersions yielding high-quality assemblies displayed a macroscopic appearance reminiscent of previously described PEG-AuNS dispersions, i.e., a bright metallic luster. ${ }^{22}$ For fixed ambient temperature and humidity, this characteristic golden shimmer was consistently observed after a composition-controlled induction period, during which the alcohol/water mixture was allowed to evaporate partially: depending on particle concentration and EtOH content (see Materials and Methods in the Supporting
Information (SI)), the drying droplets changed their appearance from dark brown to golden within seconds to minutes when AuNRs initiated pre-assembly at the air/liquid interface, as illustrated in Figure $2 \mathrm{a}^{41}$ This pre-assembly process, not observed in purely aqueous dispersions, was found to consistently start at the droplet rim, i.e., the area of highest curvature and drying speed, indicating that the process is driven by convection due to EtOH evaporation. ${ }^{42}$ The macroscopic appearance of a droplet (insets of Figure $2 b-d$ ) at selected stages of evaporation can be correlated with the resulting mesostructures, as revealed by subsequent SEM measurements exemplified in Figure $2 b-d$. A clear relationship between the aggregation state of AuNRs at the air/liquid interface at the moment of contacting the substrate and the final particle distribution after drying was established: at short pre-assembly times, most of the liquid was found to move toward the nonpatterned rim of the PDMS stamp/substrate sandwich, where the particles would accumulate to form the characteristic coffee ring. The opposite trend was observed for long pre-assembly times, where extended particle aggregation led to overfilling of the patterned central region. Slight particle agglomeration turned out to be most advantageous, since medium-sized lamellar AuNR clusters possess a moderate hydrodynamic mobility and cannot easily migrate to the rim or accumulate at the center of the substrate/stamp sandwich (Figure S4). ${ }^{43}$ In general, the quality of the local array structure and the overall macroscopic surface homogeneity were optimal when the substrates were placed onto the stamp just before the surface of the droplets turned golden (see the SI: Materials and Methods and Figure S5).

A detailed kinetic study of the process for varying composition of the dispersion medium is summarized in Figure 3, which shows that an increase in the initial EtOH/ $\mathrm{H}_{2} \mathrm{O}$ ratio has an effect similar to a reduced duration of the evaporation period. Four different morphologies can be distinguished, which are marked with frames of different
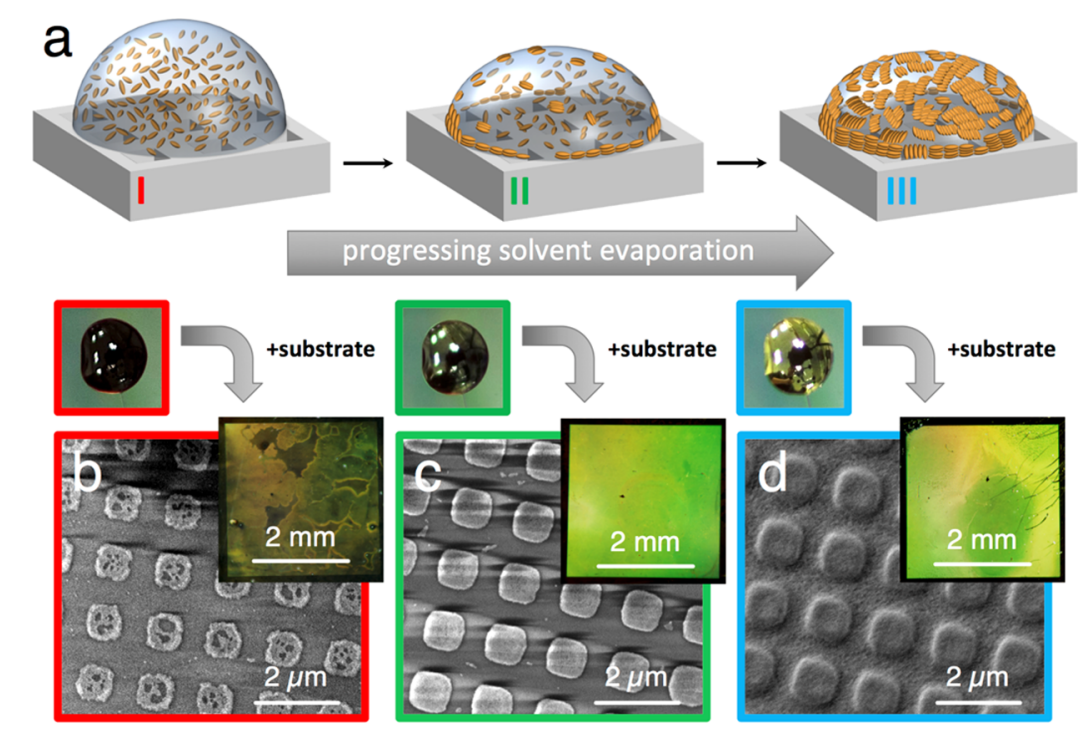

Figure 2. Influence of pre-evaporation and correlation between droplet appearance and the resulting assembly structure. Partial evaporation of $\mathrm{EtOH}$ leads to the pre-assembly of the AuNRs at the liquid-air interface, causing a golden shimmer at the droplet surface due to interparticle coupling ( $\mathrm{a}$ and inset droplet photos). For identical initial composition and volume of the dispersion, contact with the target substrate at different times leads to distinct substrate qualities $(b-d)$. The degree of pre-assembly directly correlates with the amount of particles deposited on the structured substrate area, where the underfilled surface is marked with a red frame, the optimally prepared sample with a green frame, and a vastly overfilled array with a blue one. 

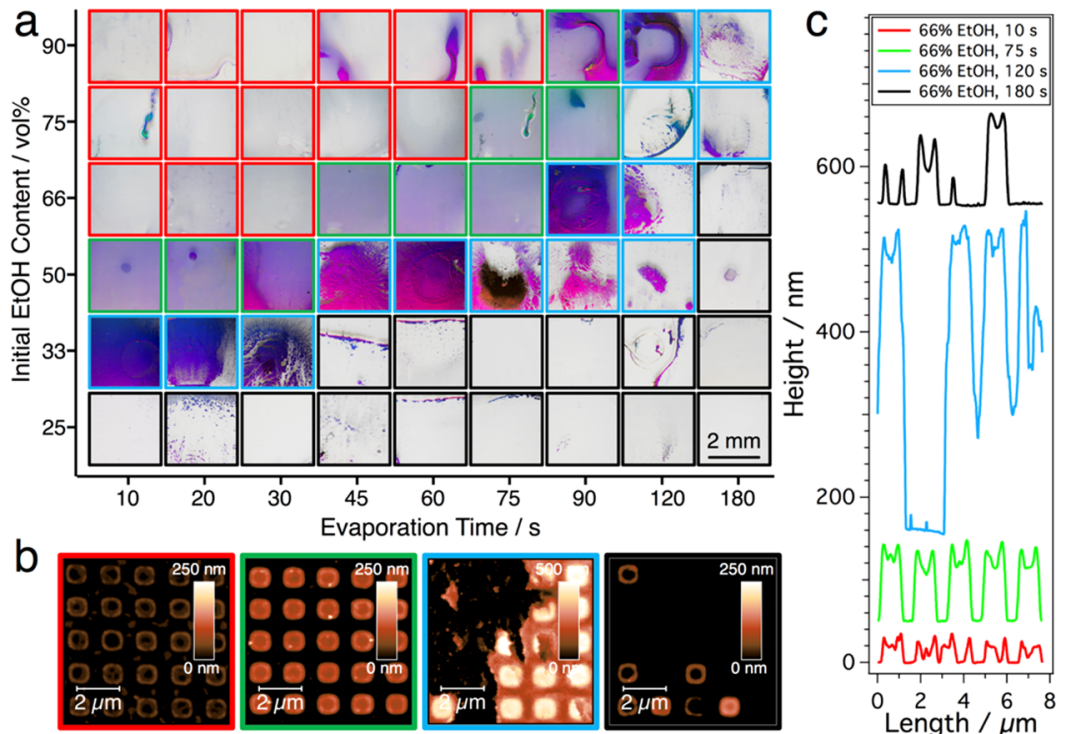

Figure 3. Influence of initial EtOH content and evaporation time prior to contact with the substrate (particle concentration constant @100 mM $\mathrm{Au}^{0}$ ). Four different substrate qualities can be distinguished, marked with colored frames in (a). EtOH concentrations below 33 vol $\%$ always yielded empty substrate centers. Dispersions with higher alcohol content produced very homogeneous arrays (green), with correct timing. To avoid underfilling (red), longer waiting times were required for higher initial EtOH concentrations, whereby excessive solvent evaporation leads to particle accumulation at the substrate center (blue) in all series. AFM height images in (b) for substrates from the series with an initial EtOH content of $66 \mathrm{vol} \%$ correspond to evaporation times of 10, 75, 120, and $180 \mathrm{~s}$ (from left to right). The cross sections in (c) were measured for the bottom row of the corresponding square arrays in the AFM images and plotted with an offset in the $z$-direction for the sake of clarity.

colors: apart from the above discussed three scenarios, namely "underfilled" (red frame), "optimally filled" (green frame), and "overfilled" (blue frame), we found that for long evaporation periods or very low initial $\mathrm{EtOH}$ content, nearly empty substrate centers (black frame) were obtained. This emergence of a dominant coffee ring effect can be attributed to the redispersion of agglomerated particles after the evaporation of a major fraction of added EtOH (Figure S4).

With this effect in mind, we investigated whether the optimum time for contact with the substrate was determined by the $\mathrm{EtOH} / \mathrm{H}_{2} \mathrm{O}$ ratio at the moment of substrate contact (i.e., independent of the concentration of dispersed particles), or by the amount of pre-assembled particle clusters at the droplet surface (i.e., strongly dependent on particle concentration in the dispersion). As shown in Figure 4 for fixed waiting time and initial EtOH$/ \mathrm{H}_{2} \mathrm{O}$ ratio, increasing the particle concentration also lead to particle accumulation at the substrate centers (see Figure S6 for macroscopic characterization). We therefore conclude that the outcome of the assembly process is mainly determined by the state of particle agglomeration at the liquid-air interface at the moment of contact with the substrate.

To better understand the influence of EtOH on the assembly behavior of the AuNRs, the molecular interactions of MUHEG-coated Au surfaces in contact with the solvent were simulated using all-atom molecular dynamics. These simulations reproduced the environment of the AuNRs in the real dispersions, where $\mathrm{CTAB}$ was present at concentrations ranging from 30 to $500 \mu \mathrm{M}$. The equilibrated system shows that some $\mathrm{CTA}^{+}$molecules reside within the MUHEG layer with their quaternary ammonium headgroups oriented toward the $\mathrm{Au}$ surface, whereas $\mathrm{CTAB}$ micelles form and reside at the MUHEG-water interface (Figure S7). In pure water, the $\mathrm{CTAB}$ micelle remained bound at the interface of the MUHEG layer for the entire duration of the simulation,

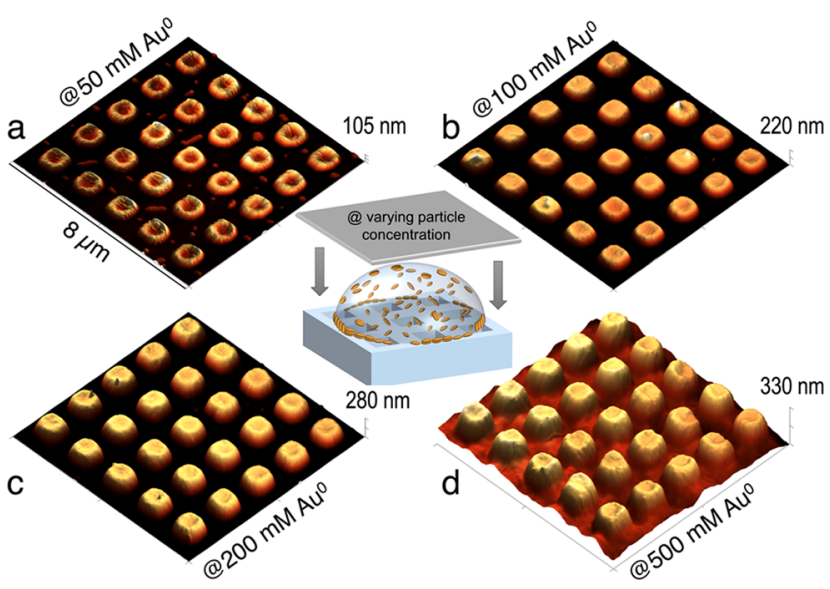

Figure 4. Influence of the particle concentration on array height and homogeneity. (a-d) AFM height images of representative arrays prepared with identical initial EtOH concentration, timing of substrate application, and surfactant concentration. Particle concentrations were varied within the range $\left[\mathrm{Au}^{0}\right]=50-500 \mathrm{mM}$. From (a) to (c), the height of the separated AuNR posts gradually increased, until a film of connected posts was obtained at the highest concentration (d).

which is attributed to minimization of the free energy by reducing the amount of interfacial water (Figure S7b). Following $200 \mathrm{~ns}$ of simulation in pure water, water molecules were removed from the system and replaced with either pure $\mathrm{EtOH}$ (Figure S8) or 52\% (v/v) EtOH in water (Figure 5). As demonstrated by the timeline of the simulation trajectories, the introduction of $\mathrm{EtOH}$ results in a quick dissociation of the $\mathrm{CTAB}$ micelle from the MUHEG interface, even in a mixture with water (Figures 5a and S9).

Regardless of the initial position of the CTAB micelle at the MUHEG/solvent interface, dissociation of the micelle from the interface could be clearly observed after $100 \mathrm{~ns}$, both in 


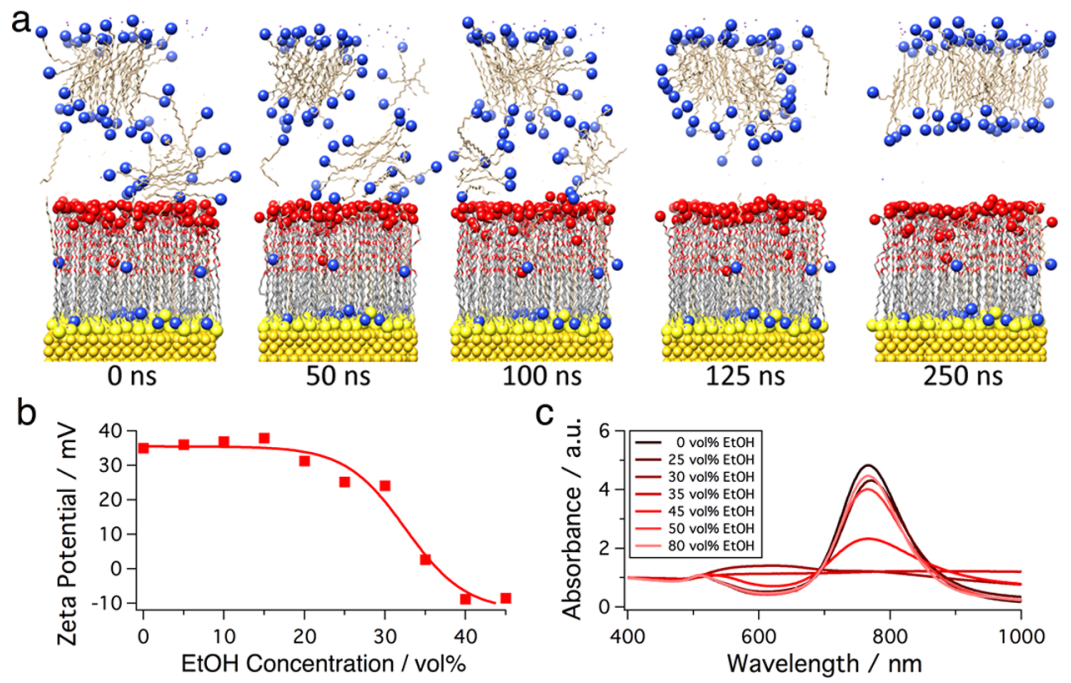

Figure 5. All-atom molecular dynamics simulations of CTAB micelles interacting with a MUHEG-functionalized Au(100) surface (91 MUHEG and $71 \mathrm{CTAB}$ molecules in $52 \%(\mathrm{v} / \mathrm{v}) \mathrm{EtOH})$ and experimental verification. Initial atom coordinates in (a) are derived from a separate $100 \mathrm{~ns}$ simulation in pure water. The originally bound surfactant micelle completely detaches from the MUHEG ligand layer within $125 \mathrm{~ns}$ after switching the solvent from pure water to an EtOH-containing mixture. (b) Addition of EtOH to a particle dispersion $([\mathrm{CTAB}]=500 \mu \mathrm{M})$ causes a strong decay of the $\zeta$-potential above $25 \%(\mathrm{v} / \mathrm{v})$ of alcohol. At 35\% (v/v) EtOH, surface-bound surfactant micelles are completely detached, leaving the particle surface uncharged. (c) In UV-vis spectroscopy measurements, fast agglomeration of the AuNRs at intermediate EtOH concentrations of $30-45 \%(\mathrm{v} / \mathrm{v})$ becomes obvious.

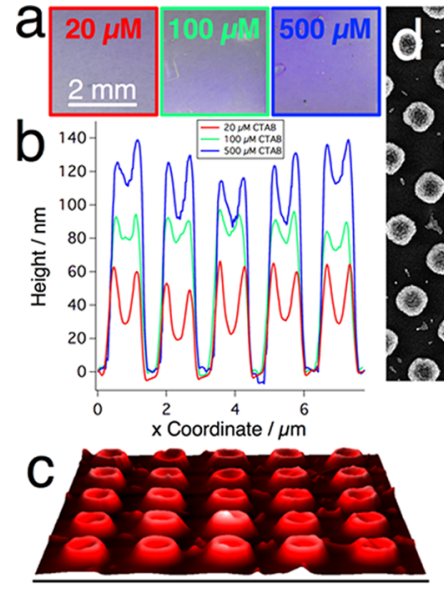

$8 \mu \mathrm{m}$

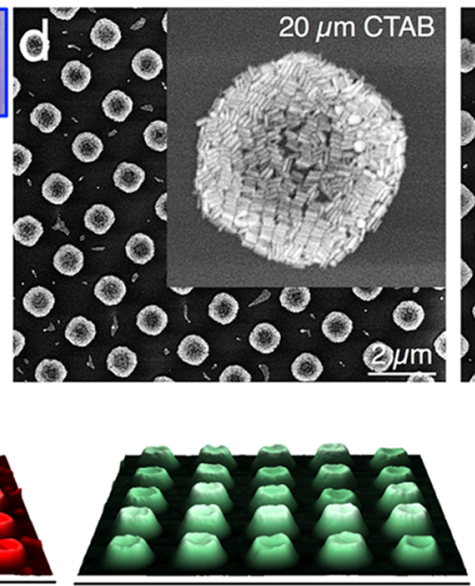

$8 \mu \mathrm{m}$

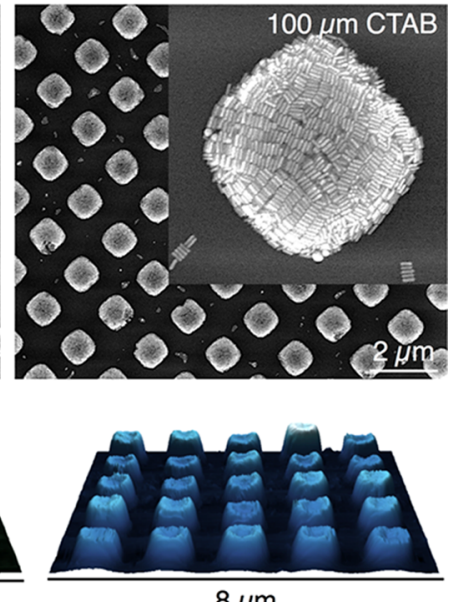

$8 \mu \mathrm{m}$

Figure 6. Influence of $\mathrm{CTAB}$ surfactant concentration $(20-500 \mu \mathrm{m})$. (a) Photographs of three substrates obtained at different [CTAB], revealing similar macroscopic homogeneity. (b) Cross sections measured from AFM height images (c), which show increasing average post height with surfactant concentration in the dispersion, and the presence of an elevated rim in all posts. (d) SEM micrographs for low and intermediate CTAB concentrations demonstrate that complete filling is only reached above a minimum surfactant concentration.

pure $\mathrm{EtOH}$ and in the water/EtOH mixture (Figures 5a and S8). Visual observations are confirmed by the radial distribution function of the quaternary ammonium headgroup of $\mathrm{CTA}^{+}$interacting with the terminal $-\mathrm{OH}$ group of MUHEG, which was significantly decreased by the end of simulations in both EtOH and the water/EtOH mixture (Figure S9b,d). The electron density of the system over the simulation trajectory also exhibits the dissociation of the CTAB micelle from the MUHEG/solvent interface (Figure S9a,c). The removal of adsorbed CTAB micelles can explain both the experimentally observed clustering of AuNRs and their increased affinity toward the air-liquid interface, as the result of a more hydrophobic character of the particle coating.

To verify the mechanism supported by the MD simulations, we conducted $\zeta$-potential and UV-vis spectroscopy measure- ments of AuNR colloids for varying $\mathrm{CTAB}$ and $\mathrm{EtOH}$ concentrations. In water, the particles exhibited a strongly positive $\zeta$-potential, even far below the cmc of CTAB (e.g., +35 $\mathrm{mV}$ above $100 \mu \mathrm{M}$; Figure S10). With EtOH contents above $25 \%(\mathrm{v} / \mathrm{v})$, however, the surface charge dropped, reaching $0 \mathrm{mV}$ at $35 \%(\mathrm{v} / \mathrm{v})$ and $-10 \mathrm{mV}$ at $45 \%(\mathrm{v} / \mathrm{v}) \mathrm{EtOH}$ (Figure $5 \mathrm{~b}$ ). Whereas AuNRs were prepared and stored at $\mathrm{CTAB}$ concentrations above the $\mathrm{cmc}(1 \mathrm{mM}), \zeta$-potential measurements were conducted after dilution, well below the cmc $(30 \mu \mathrm{M} \leq[\mathrm{CTAB}] \leq 500 \mu \mathrm{M})$. These data support the hypothesis that, in water, $\mathrm{CTAB}$ micelles remain bound to the AuNR-MUHEG interface far below the $\mathrm{cmc}([\mathrm{CTAB}] \geq 30$ $\mu \mathrm{M})$ but are readily removed by EtOH. The resulting loss of colloidal stability is mirrored in the UV-vis spectra (Figure 5c) showing peaks around $600 \mathrm{~nm}$ that are characteristic for 
side-to-side aggregation of AuNRs. ${ }^{44}$ Collectively, these results make a strong case for the idea that $\mathrm{EtOH}$ efficiently triggers the agglomeration and surface activity of AuNRs, thereby reducing particle mobility during drying and ultimately leading to a strongly altered self-assembly behavior. Similar trapping of nanoparticles at the liquid-air interface has been recognized as a method for reducing coffee stains and building highly ordered close-packed layers. ${ }^{41}$

Interestingly, the surfactant concentration itself influences the structure of self-assembled AuNR arrays in a counterintuitive manner. Whereas for dispersions in pure water the quality of the assembly deteriorated when increasing the surfactant concentration, EtOH-containing dispersions were less influenced by this parameter, as $\mathrm{CTAB}$ concentrations up to $500 \mu \mathrm{M}$ did not significantly alter the homogeneity and array dimensions. However, the concentration of free surfactant did influence the average height of the individual posts, leading to roughly 3 -fold higher structures when $\mathrm{CTAB}$ concentration was increased from 20 up to $500 \mu \mathrm{M}$ (Figure 6). As the volume occupied by $\mathrm{CTAB}$ is negligible compared to the volume of gold in all cases (around $100 \mu \mathrm{M}$ CTAB vs 100 $\mathrm{mM} \mathrm{Au}{ }^{0}$ ), such height differences must be caused by varying amounts of particles being deposited outside the patterned stamp area.

Potential explanations for this behavior are changes of the stamp wetting and meniscus shape during late drying stages, or the interplay of solute- and surfactant-driven Marangoni flows that influence particle repartition. ${ }^{4,45-47}$ Although this behavior beckons a separate in-depth investigation, we further explore the efficacy of substrates produced by this assembly approach for molecular ultradetection. As shown in Figure 7, this kinetically controlled assembly technique paves the way to highly regular AuNR arrays with feature sizes ranging from 200 $\mathrm{nm}$ up to tens of microns.

In a recent work, we demonstrated that arrangement of gold nanoparticles into periodic microarrays can significantly boost SERS sensitivity via tailored lattice plasmon resonances. ${ }^{22}$ Such lattice plasmon resonances typically occur near a Rayleigh anomaly, i.e., a strong change of reflectivity due to diffraction at the metal grating. ${ }^{48}$ For square arrays of gold nanoparticles, the lattice resonance wavelength can be approximated as $\lambda_{\max }=$ $L n_{\text {subs, }}$, where the lattice parameter $L$ corresponds to the centerto-center distance between neighboring posts and $n_{\text {subs }}$ to the refractive index of the substrate. ${ }^{49,50}$ Shown in Figure 7 are AuNR arrays with $L$ ranging from $20 \mu \mathrm{m}$ down to $400 \mathrm{~nm}$. The corresponding extinction spectra (Figure S11) show that for each lattice parameter, distinct resonance peaks arise near the predicted position of the Rayleigh anomaly. Exploitation of these lattice resonances in SERS spectroscopy demands precise matching with the wavelength of the excitation laser and therefore requires highly regular arrays with submicrometer periodicities for the typically employed UV-vis/NIR Raman setups. ${ }^{2,51}$

To assess these substrates in a practical sensing application, we chose the bacterial pigment/toxin pyocyanin as a probe molecule. Pyocyanin is a Raman-active quorum-sensing molecule expressed by $P$. aeruginosa, which can be detected by SERS using NIR excitation. ${ }^{25}$ The SERS spectra of pyocyanin in water and Luria-Bertrani (LB) bacterial growth medium were recorded using patterned substrates with lattice parameters between $400 \mathrm{~nm}$ and $1.66 \mu \mathrm{m}$ (corresponding to feature dimensions between $200 \mathrm{~nm}$ and $1 \mu \mathrm{m}$; see SI Table $\mathrm{S} 1$ ), as shown in Figure 8. A $785 \mathrm{~nm}$ laser was chosen as the
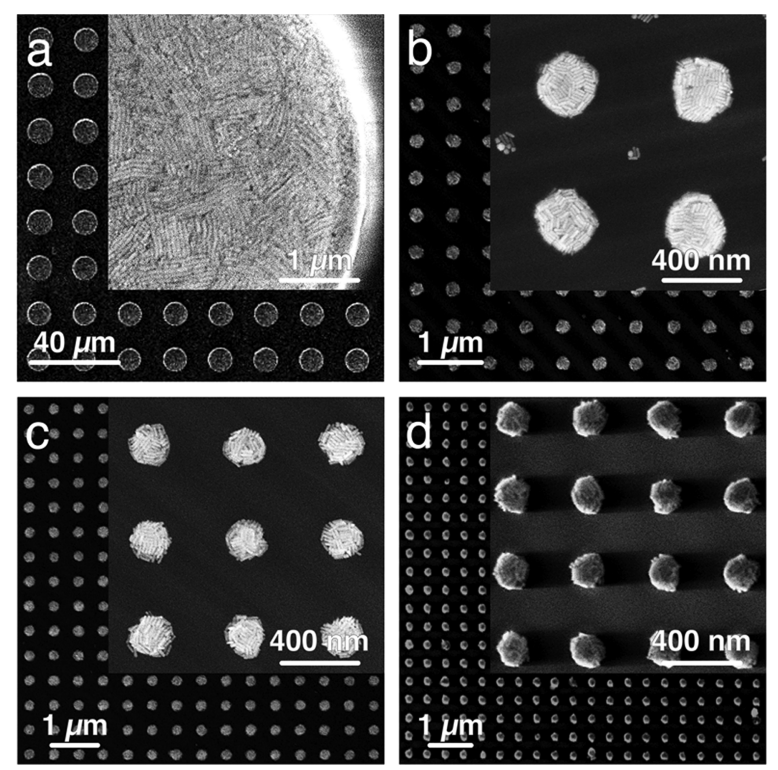

Figure 7. Feature size variation from $10 \mu \mathrm{m}$ to $200 \mathrm{~nm}$ (lattice parameters from $(\mathrm{a}-\mathrm{d}): 20 \mu \mathrm{m}, 600,500$, and $400 \mathrm{~nm})$. SEM images show that tall, micron-sized structures (a) exhibit the best array quality when prepared with high particle concentrations (up to 850 $\mathrm{mM} \mathrm{Au}{ }^{0}$ ) and very short induction periods (below $15 \mathrm{~s}$ ), whereas submicrometer structures $(b-d)$ exhibit good filling for much lower particle concentrations (around $100 \mathrm{mM} \mathrm{Au}{ }^{0}$ ) when combined with intermediate pre-assembly times (around $45 \mathrm{~s}$ ). For all samples, a side-by-side arrangement of AuNRs could be confirmed by highresolution SEM images (insets). For large structures in (a), parallel lamellae of lying rods spanning over several micrometers were found.

excitation source because it targets both the first transparency window of biological systems and the excitation wavelength of the analyte molecule. In fact, this laser is the most widespread light source for Raman and other optical measurements in biological media due to its relatively low tendency to cause photoluminescence emission overlaying the signals. ${ }^{22}$ With the excitation at $785 \mathrm{~nm}$, one would expect the highest signal enhancement for a lattice parameter $L=500 \mathrm{~nm}$, for which a resonance around $770 \mathrm{~nm}$ is predicted by the formula for $\lambda_{\max }$ above.

Compared to arrays with micrometer-sized lattices, the arrangements with submicrometer lattice parameters indeed give rise to significantly enhanced SERS: in Figure 8a, the SERS spectrum of pyocyanin in water recorded on a $1.66 \mu \mathrm{m}$ lattice shows the dominant Raman modes (1352 and $\sim 1600$ $\mathrm{cm}^{-1}$ ), with a meager signal of $\sim 1000$ counts/s, whereas the miniaturized arrays with lattice parameters of 400 and $500 \mathrm{~nm}$ have close to 25 -fold intensity. Plotting the signal intensity of the dominant mode at $1600 \mathrm{~cm}^{-1}$ (from phenyl ring deformation and $\mathrm{C}-\mathrm{C}$ stretching) against the lattice parameter (Figure $8 \mathrm{~b}$ ) clearly reveals an inverse correlation of feature size and SERS intensity. As all the probed substrates have a very similar percentage of effective area covered with nanoparticles $(25-27 \%)$, the observed trends must arise from the different lattice parameters and increasing surface-to-volume ratios with decreasing feature size. Figure $8 \mathrm{c}$ displays the dependence of the SERS signal at $1600 \mathrm{~cm}^{-1}$ on the pyocyanin concentration in water, for the best substrate (shown in Figure $7 \mathrm{~d}$ ), suggesting a detection limit of approximately $10^{-12} \mathrm{M}$.

For SERS-based clinical diagnostics, measuring in complex biofluids represents a significant challenge due to fouling of the 

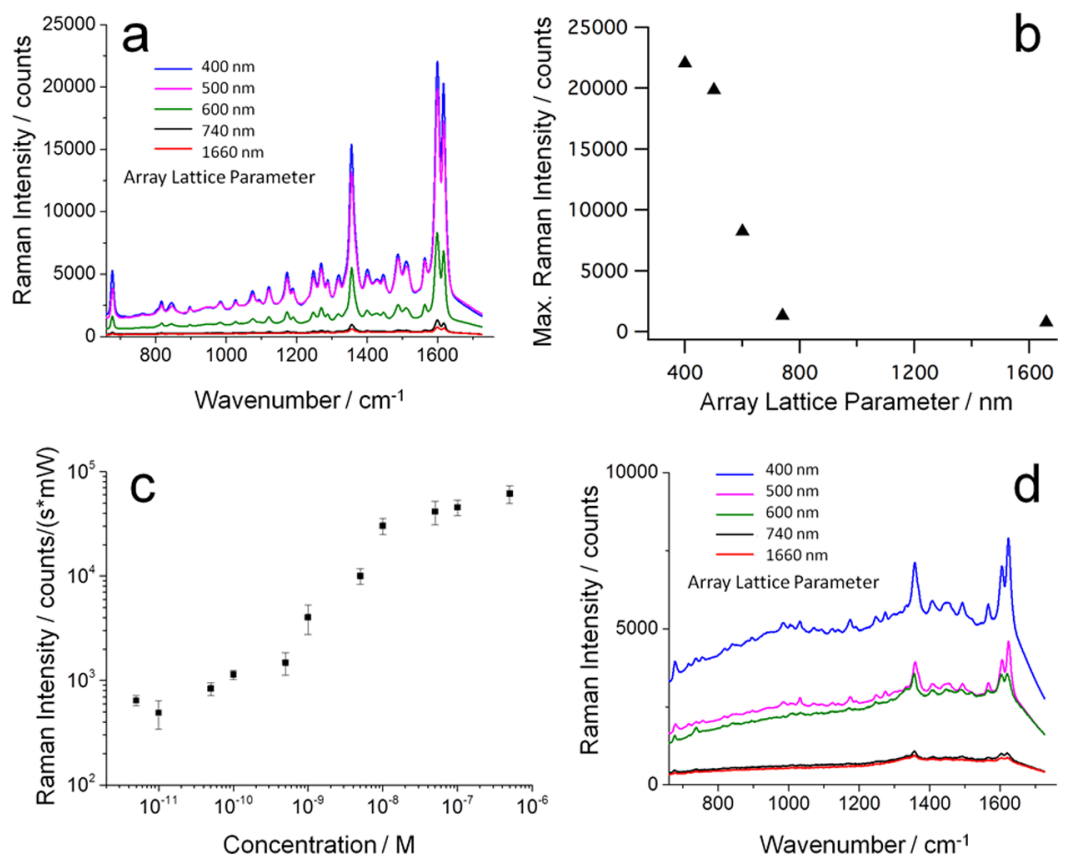

Figure 8. SERS spectroscopy of pyocyanin using hierarchically assembled gold nanoparticle arrays and $785 \mathrm{~nm}$ excitation. (a) SERS spectra of 1 $\mu \mathrm{m}$ pyocyanin in water, recorded on periodic square arrays with varying lattice parameter. Strongly improved signals are observed for submicrometer lattice arrays. The corresponding SERS intensity of the $1600 \mathrm{~cm}^{-1}$ peak rises steeply with decreasing lattice parameter below a critical value of $L=740 \mathrm{~nm}$ (b). (c) The normalized SERS intensity of $1 \mu \mathrm{M}$ to $1 \mathrm{pM}$ pyocyanin on the champion substrate displays a sigmoidal shape, where the SERS signal undergoes a 100 -fold increase between 0.1 and $10 \mathrm{nM}$ pyocyanin. (d) SERS spectra from pyocyanin in LB broth prove efficient detection in complex media. The spectra are the average from multiple measurements $(n=50-150)$ and measured with a laser power of $1.5 \mathrm{~mW}$. The integration time was $1 \mathrm{~s}$ for all spectra in (a) and $5 \mathrm{~s}$ for the data shown in (d).

plasmonic metal surface with biomolecules, which restricts the access of the analyte to the surface and thus reduces signal enhancement. In a recent study, we showed that micron-scale arrays of gold nanoparticles allowed ultrasensitive in situ detection of pyocyanin in cultures of $P$. aeruginosa, when coated with mesoporous silica. The mesoporous layer acted as a sieve to prevent larger proteins and biomolecules from fouling the gold nanoparticle interface while allowing the percolation and detection of small molecules down to femtomolar concentrations. ${ }^{25}$ In another recent study, a nacre-like film impregnated with AuNPs exhibited a similar increase in signal due to filtration by the porous polymer network compared to a film of bare AuNPs. ${ }^{52}$ Intriguingly, we observe that although the lack of such a filtration layer leads to a moderate signal background from the adsorption of biomolecules like peptides and yeast tryptone, the submicrometer patterned substrates introduced in this work still yield very clear SERS spectra in biological media (Figure 8d).

\section{CONCLUSIONS}

A kinetically controlled approach for the template-assisted assembly of plasmonic nanoparticles, which relies on the addition of alcohol to aqueous AuNP dispersions, is reported herein. This approach largely improves the large-scale reproducibility and overall quality of the resulting arrays. As the crucial experimental parameters in this method, we identified the dispersion medium composition, solvent evaporation time, particle concentration, and surfactant concentration. A strong correlation was found between particle pre-assembly at the droplet-air interface and quality of the resulting final substrate. Through a combination of experiments and simulations, the underlying mechanism for this effect was studied, supporting the idea that the removal of residual surfactant micelles from the interface of the MUHEGcoating on the particles plays a crucial role in the self-assembly behavior. The resulting colloidal destabilization leads to enhanced surface activity and partial agglomeration of the gold nanoparticles, which turned out to be advantageous for creating homogeneous AuNR arrays over $\mathrm{mm}^{2}$ areas. This method enables the routine fabrication of high-quality periodic arrays with submicrometer features and was found to be easier, faster, and more reliable than common multistep methods based on spin or dip coating and subsequent transfer printing.

The resulting structures successfully combine plasmonic and photonic resonances to deliver significant improvements in SERS sensor fabrication. Consequently, they allow sensitive biomolecule detection with substantially reduced material costs and preparative effort, as demonstrated with the bacterial pathogen pyocyanin both in water and Luria-Bertrani medium. The capability of these substrates for detecting bacterial quorum-sensing molecules in bacterial growth medium, even without an additional filtration layer, should facilitate in situ studies of bacterial growth/quorum sensing via SERS and could be particularly useful for studying the effects of surface topography on bacterial attachment and biofilm formation. ${ }^{53}$ Finally, we propose that the particle assembly mechanism discussed herein is of a universal nature and could have important implications for the field of template-assisted colloidal assembly. We therefore expect this proof of concept to trigger deeper investigations utilizing nanoparticles of varied materials composition and surface functionalization, as well as the exploration of a plethora of pattern designs and target substrates. 


\section{ASSOCIATED CONTENT}

\section{S Supporting Information}

The Supporting Information is available free of charge on the ACS Publications website at DOI: 10.1021/acsami.9b00334.

Experimental part; characterization of nanoparticles and large area substrates by electron microscopy; microscopic investigation of an evaporating AuNR dispersion droplet; study of the impact of evaporation time and particle concentration on macroscopic substrate homogeneity; additional simulations of the interaction of CTAB with MUHEG-coated AuNRs in water and EtOH; UV-vis and $\zeta$-potential data of MUHEGAuNRs with varying CTAB concentration; optical characterization of the substrates by UV-vis-NIR spectroscopy (PDF)

\section{AUTHOR INFORMATION}

\section{Corresponding Authors}

*E-mail: christoph.hanske@googlemail.com (C.H.).

*E-mail: 1lizmarzan@cicbiomagune.es (L.M.L.-M.).

\section{ORCID $\odot$}

Agustín Mihi: 0000-0003-3821-7881

Luis M. Liz-Marzán: 0000-0002-6647-1353

\section{Present Address}

"Institute of Advanced Ceramics, Hamburg University of Technology, 21073 Hamburg, Germany (E.H.H.).

\section{Notes}

The authors declare no competing financial interest.

\section{ACKNOWLEDGMENTS}

This work has been funded by the Spanish MINECO (Grant No. MAT2017-86659-R). C.H. acknowledges the Alexander von Humboldt Foundation for funding in the framework of a Feodor Lynen fellowship. E.H.H. acknowledges the receipt of a Juan de la Cierva-fellowship (FJCI-2014-22598). A.M. is grateful to the funding from the European Research Council (ERC) under the European Union's Horizon 2020 research and innovation program (grant agreement No. 637116, ENLIGHTMENT) and funding by the Spanish Ministerio de Economía, Industria y Competitividad (MINECO) (Grant Nos. SEV-2015-0496 and MAT2016-79053-P). The authors further thank Mathias Charconnet for fruitful discussions and Vished Kumar for TEM imaging. This work was performed under the Maria de Maeztu Units of Excellence Program from the Spanish State Research Agency (Grant No. MDM-20170720). We acknowledge support of the publication fee by the CSIC Open Access Publication Support Initiative through its Unit of Information Resources for Research (URICI).

\section{REFERENCES}

(1) Knight, M. W.; Sobhani, H.; Nordlander, P.; Halas, N. J. Photodetection with Active Optical Antennas. Science 2011, 332, 702-704.

(2) Yan, B.; Boriskina, S. V.; Reinhard, B. M. Design and Implementation of Noble Metal Nanoparticle Cluster Arrays for Plasmon Enhanced Biosensing. J. Phys. Chem. C 2011, 115, 2443724453.

(3) Bagheri, S.; Weber, K.; Gissibl, T.; Weiss, T.; Neubrech, F.; Giessen, H. Fabrication of Square-Centimeter Plasmonic Nanoantenna Arrays by Femtosecond Direct Laser Writing Lithography: Effects of Collective Excitations on SEIRA Enhancement. ACS Photonics 2015, 2, 779-786.
(4) Karg, M.; König, T. A. F.; Retsch, M.; Stelling, C.; Reichstein, P. M.; Honold, T.; Thelakkat, M.; Fery, A. Colloidal Self-Assembly Concepts for Light Management in Photovoltaics. Mater. Today 2015, $18,185-205$.

(5) Chen, T.; Reinhard, B. M. Assembling Color on the Nanoscale: Multichromatic Switchable Pixels From Plasmonic Atoms and Molecules. Adv. Mater. 2016, 28, 3522-3527.

(6) Pastoriza-Santos, I.; Kinnear, C.; Perez-Juste, J.; Mulvaney, P.; Liz-Marzan, L. M. Plasmonic Polymer Nanocomposites. Nat. Rev. Mater. 2018, 3, 375-391.

(7) Kraus, T.; Brodoceanu, D.; Pazos-Pérez, N.; Fery, A. Colloidal Surface Assemblies: Nanotechnology Meets Bioinspiration. Adv. Funct. Mater. 2013, 23, 4529-4541.

(8) Schlücker, S. Surface-Enhanced Raman Spectroscopy: Concepts and Chemical Applications. Angew. Chem., Int. Ed. 2014, 53, 47564795.

(9) Liu, N.; Mesch, M.; Weiss, T.; Hentschel, M.; Giessen, H. Infrared Perfect Absorber and Its Application as Plasmonic Sensor. Nano Lett. 2010, 10, 2342-2348.

(10) Petefish, J. W.; Hillier, A. C. Angle-Tunable Enhanced Infrared Reflection Absorption Spectroscopy via Grating-Coupled Surface Plasmon Resonance. Anal. Chem. 2014, 86, 2610-2617.

(11) Ye, X.; Zheng, C.; Chen, J.; Gao, Y.; Murray, C. B. Using Binary Surfactant Mixtures to Simultaneously Improve the Dimensional Tunability and Monodispersity in the Seeded Growth of Gold Nanorods. Nano Lett. 2013, 13, 765-771.

(12) Link, S.; El-Sayed, M. A. Shape and Size Dependence of Radiative, Non-Radiative and Photothermal Properties of Gold Nanocrystals. Int. Rev. Phys. Chem. 2000, 19, 409-453.

(13) González-Rubio, G.; Díaz-Núñez, P.; Rivera, A.; Prada, A.; Tardajos, G.; González-Izquierdo, J.; Bañares, L.; Llombart, P.; Macdowell, L. G.; Alcolea Palafox, M.; Liz-Marzán, L. M.; PeñaRodríguez, O.; Guerrero-Martínez, A. Femtosecond Laser Reshaping Yields Gold Nanorods with Ultranarrow Surface Plasmon Resonances. Science 2017, 358, 640-644.

(14) Camden, J. P.; Dieringer, J. A.; Wang, Y.; Masiello, D. J.; Marks, L. D.; Schatz, G. C.; Van Duyne, R. P. Probing the Structure of SingleMolecule Surface-Enhanced Raman Scattering Hot Spots. J. Am. Chem. Soc. 2008, 130, 12616-12617.

(15) Slaughter, L. S.; Wang, L.-Y.; Willingham, B. A.; Olson, J. M.; Swanglap, P.; Dominguez-Medina, S.; Link, S. Plasmonic Polymers Unraveled Through Single Particle Spectroscopy. Nanoscale 2014, 6, 11451-11461.

(16) Hanske, C.; Tebbe, M.; Kuttner, C.; Bieber, V.; Tsukruk, V. V.; Chanana, M.; König, T. A. F.; Fery, A. Strongly Coupled Plasmonic Modes on Macroscopic Areas via Template-Assisted Colloidal SelfAssembly. Nano Lett. 2014, 14, 6863-6871.

(17) Tian, C.; Deng, Y.; Zhao, D.; Fang, J. Plasmonic Silver Supercrystals with Ultrasmall Nanogaps for Ultrasensitive SERSBased Molecule Detection. Adv. Opt. Mater. 2015, 3, 404-411.

(18) Kang, M.; Park, S.-G.; Jeong, K.-H. Repeated Solid-state Dewetting of Thin Gold Films for Nanogap-Rich Plasmonic Nanoislands. Sci. Rep. 2015, 5, No. 14790.

(19) Bruchhaus, L.; Mazarov, P.; Bischoff, L.; Gierak, J.; Wieck, A. D.; Hövel, H. Comparison of Technologies for Nano Device Prototyping with a Special Focus on Ion Beams: a Review. Appl. Phys. Rev. 2017, 4, No. 011302.

(20) Wang, D.; Yang, A.; Wang, W.; Hua, Y.; Schaller, R. D.; Schatz, G. C.; Odom, T. W. Band-Edge Engineering for Controlled MultiModal Nanolasing in Plasmonic Superlattices. Nat. Nanotechnol. 2017, 12, 889-894.

(21) Cui, Y.; Björk, M. T.; Liddle, J. A.; Sönnichsen, C.; Boussert, B.; Alivisatos, A. P. Integration of Colloidal Nanocrystals Into Lithographically Patterned Devices. Nano Lett. 2004, 4, 1093-1098.

(22) Matricardi, C.; Hanske, C.; Garcia-Pomar, J. L.; Langer, J.; Mihi, A.; Liz-Marzan, L. M. Gold Nanoparticle Plasmonic Superlattices as Surface-Enhanced Raman Spectroscopy Substrates. ACS Nano 2018, 12, 8531-8539. 
(23) Hanske, C.; Sanz-Ortiz, M. N.; Liz-Marzan, L. M. Silica-Coated Plasmonic Metal Nanoparticles in Action. Adv. Mater. 2018, 30, No. 1707003.

(24) Alvarez-Puebla, R. A.; Agarwal, A.; Manna, P.; Khanal, B. P.; Aldeanueva-Potel, P.; Carbó-Argibay, E.; Pazos-Pérez, N.; Vigderman, L.; Zubarev, E. R.; Kotov, N. A.; Liz-Marzán, L. M. Gold Nanorods 3D-Supercrystals as Surface Enhanced Raman Scattering Spectroscopy Substrates for the Rapid Detection of Scrambled Prions. Proc. Natl. Acad. Sci. U.S.A. 2011, 108, 8157-8161.

(25) Bodelón, G.; Montes-García, V.; López-Puente, V.; Hill, E. H.; Hamon, C.; Sanz-Ortiz, M. N.; Rodal-Cedeira, S.; Costas, C.; Celiksoy, S.; Perez-Juste, J.; Pastoriza-Santos, I.; Liz-Marzán, L. M.; et al. Detection and Imaging of Quorum Sensing in Pseudomonas aeruginosa Biofilm Communities by Surface-Enhanced Resonance Raman Scattering. Nat. Mater. 2016, 15, 1203-1211.

(26) Yan, B.; Thubagere, A.; Premasiri, W. R.; Ziegler, L. D.; Dal Negro, L.; Reinhard, B. M. Engineered SERS Substrates with Multiscale Signal Enhancement: Nanoparticle Cluster Arrays. ACS Nano 2009, 3, 1190-1202.

(27) Hamon, C.; Novikov, S.; Scarabelli, L.; Basabe-Desmonts, L.; Liz-Marzán, L. M. Hierarchical Self-Assembly of Gold Nanoparticles Into Patterned Plasmonic Nanostructures. ACS Nano 2014, 8, 10694-10703.

(28) Horn, A.; Schoberth, H. G.; Hiltl, S.; Chiche, A.; Wang, Q.; Schweikart, A.; Fery, A.; Böker, A. Nanostructured Wrinkled Surfaces for Templating Bionanoparticles-Controlling and Quantifying the Degree of Order. Faraday Discuss. 2009, 143, 143-150.

(29) Tebbe, M.; Mayer, M.; Glatz, B. A.; Hanske, C.; Probst, P. T.; Müller, M. B.; Karg, M.; Chanana, M.; König, T. A. F.; Kuttner, C.; Fery, A. Optically Anisotropic Substrates via Wrinkle-Assisted Convective Assembly of Gold Nanorods on Macroscopic Areas. Faraday Discuss. 2015, 181, 243-260.

(30) Kuemin, C.; Nowack, L.; Bozano, L.; Spencer, N. D.; Wolf, H. Oriented Assembly of Gold Nanorods on the Single-Particle Level. Adv. Funct. Mater. 2012, 22, 702-708.

(31) Flauraud, V.; Mastrangeli, M.; Bernasconi, G. D.; Butet, J.; Alexander, D. T. L.; Shahrabi, E.; Martin, O. J. F.; Brugger, J. Nanoscale Topographical Control of Capillary Assembly of Nanoparticles. Nat. Nanotechnol. 2017, 12, 73-80.

(32) Zhang, H.; Cadusch, J.; Kinnear, C.; James, T.; Roberts, A.; Mulvaney, P. Direct Assembly of Large Area Nanoparticle Arrays. ACS Nano 2018, 12, 7529-7537.

(33) Zheng, Y.; Zhong, X.; Li, Z.; Xia, Y. Successive, Seed-Mediated Growth for the Synthesis of Single-Crystal Gold Nanospheres with Uniform Diameters Controlled in the Range of 5-150 Nm. Part. Part. Syst. Charact. 2014, 31, 266-273.

(34) O’Brien, M. N.; Jones, M. R.; Brown, K. A.; Mirkin, C. A. Universal Noble Metal Nanoparticle Seeds Realized Through Iterative Reductive Growth and Oxidative Dissolution Reactions. J. Am. Chem. Soc. 2014, 136, 7603-7606.

(35) Scarabelli, L.; Sánchez-Iglesias, A.; Perez-Juste, J.; Liz-Marzán, L. M. A "Tips and Tricks" Practical Guide to the Synthesis of Gold Nanorods. J. Phys. Chem. Lett. 2015, 6, 4270-4279.

(36) Qin, D.; Xia, Y.; Whitesides, G. M. Soft Lithography for Microand Nanoscale Patterning. Nat. Protoc. 2010, 5, 491-502.

(37) Kraus, T.; Malaquin, L.; Schmid, H.; Riess, W.; Spencer, N. D.; Wolf, H. Nanoparticle Printing with Single-Particle Resolution. Nat. Nanotechnol. 2007, 2, 570-576.

(38) Deegan, R. D.; Bakajin, O.; Dupont, T. F.; Huber, G.; Nagel, S. R.; Witten, T. A. Capillary Flow as the Cause of Ring Stains From Dried Liquid Drops. Nature 1997, 389, 827-829.

(39) Nomura, T.; Asai, Y.; Murahashi, N.; Iwamoto, K. Formation of Spherical Micelles by the Novel Platelet Activating Factor Receptor Antagonist, E5880. Chem. Pharm. Bull. 2000, 48, 947-950.

(40) Vazquez, G.; Alvarez, E.; Navaza, J. M. Surface Tension of Alcohol Water + Water From 20 to $50{ }^{\circ} \mathrm{C}$. J. Chem. Eng. Data 1995, 40, 611-614.
(41) Bigioni, T. P.; Lin, X. M.; Nguyen, T. T.; Corwin, E. I.; Witten, T. A.; Jaeger, H. M. Kinetically Driven Self Assembly of Highly Ordered Nanoparticle Monolayers. Nat. Mater. 2006, 5, 265-270.

(42) Deegan, R. D.; Bakajin, O.; Dupont, T. F.; Huber, G.; Nagel, S. R.; Witten, T. A. Contact Line Deposits in an Evaporating Drop. Phys. Rev. E 2000, 62, 756-765.

(43) Kim, H.; Boulogne, F.; Um, E.; Jacobi, I.; Button, E.; Stone, H. A. Controlled Uniform Coating From the Interplay of Marangoni Flows and Surface-Adsorbed Macromolecules. Phys. Rev. Lett. 2016, 116, No. 124501.

(44) Funston, A. M.; Novo, C.; Davis, T. J.; Mulvaney, P. Plasmon Coupling of Gold Nanorods at Short Distances and in Different Geometries. Nano Lett. 2009, 9, 1651-1658.

(45) Karpitschka, S.; Riegler, H. Noncoalescence of Sessile Drops From Different but Miscible Liquids: Hydrodynamic Analysis of the Twin Drop Contour as a Self-Stabilizing Traveling Wave. Phys. Rev. Lett. 2012, 109, No. 066103.

(46) Li, P.; Li, Y.; Zhou, Z.-K.; Tang, S.; Yu, X.-F.; Xiao, S.; Wu, Z.; Xiao, Q.; Zhao, Y.; Wang, H.; Chu, P. K. Evaporative Self-Assembly of Gold Nanorods Into Macroscopic 3D Plasmonic Superlattice Arrays. Adv. Mater. 2016, 28, 2511-2517.

(47) Sempels, W.; De Dier, R.; Mizuno, H.; Hofkens, J.; Vermant, J. Auto-Production of Biosurfactants Reverses the Coffee Ring Effect in a Bacterial System. Nat. Commun. 2013, 4, No. 1757.

(48) Khlopin, D.; Laux, F.; Wardley, W. P.; Martin, J.; Wurtz, G. A.; Plain, J.; Bonod, N.; Zayats, A. V.; Dickson, W.; Gérard, D. Lattice Modes and Plasmonic Linewidth Engineering in Gold and Aluminum Nanoparticle Arrays. J. Opt. Soc. Am. B 2017, 34, 691-700.

(49) Guo, R.; Hakala, T. K.; Törmä, P. Geometry Dependence of Surface Lattice Resonances in Plasmonic Nanoparticle Arrays. Phys. Rev. B 2017, 95, No. 155423.

(50) Auguié, B.; Bendaña, X. M.; Barnes, W. L.; García de Abajo, F. J. Diffractive Arrays of Gold Nanoparticles Near an Interface: Critical Role of the Substrate. Phys. Rev. B 2010, 82, No. 155447.

(51) Huang, Y.; Zhang, X.; Ringe, E.; Hou, M.; Ma, L.; Zhang, Z. Tunable Lattice Coupling of Multipole Plasmon Modes and NearField Enhancement in Closely Spaced Gold Nanorod Arrays. Sci. Rep. 2016, No. 23159.

(52) Hill, E. H.; Hanske, C.; Johnson, A.; Yate, L.; Jelitto, H.; Schneider, G. A.; Liz-Marzán, L. M. Metal Nanoparticle Growth Within Clay-Polymer Nacre-Inspired Materials for Improved Catalysis and Plasmonic Detection in Complex Biofluids. Langmuir 2017, 33, 8774-8783.

(53) Hill, E. H.; Liz-Marzán, L. M. Toward Plasmonic Monitoring of Surface Effects on Bacterial Quorum-Sensing. Curr. Opin. Colloid Interface Sci. 2017, 32, 1-10. 